\title{
Efficacy and safety of anti-angiogenesis medicines for advanced soft tissue sarcoma: a meta-analysis
}

\author{
Yuxuan Wang, Xiaobo Dai, Yuxi Zhu \\ Department of Oncology, The First Affiliated Hospital of Chongqing Medical University, Chongqing 400016, China \\ Contributions: (I) Conception and design: Y Zhu, Y Wang; (II) Administrative support: Y Zhu, X Dai; (III) Provision of study materials or patients: Y \\ Wang; (IV) Collection and assembly of data: Y Wang; (V) Data analysis and interpretation: Y Wang, Y Zhu; (VI) Manuscript writing: All authors; (VII) \\ Final approval of manuscript: All authors. \\ Correspondence to: Yuxi Zhu. Department of Oncology, The First Affiliated Hospital of Chongqing Medical University, No. 1 Youyi Road, Yuzhong \\ District, Chongqing 400016, China. Email: zhuyuxi@hospital.cqmu.edu.cn.
}

\begin{abstract}
Background: Surgery-based treatment is the standard method for the early-stage soft tissue sarcoma (STS) with relatively good clinical outcome. However, the recurrence and metastasis decrease the survival of STS, regardless of surgery, radiotherapy, and chemotherapy. On the other hand, anti-angiogenesis treatments for STS have been clinically applied in recent years. To evaluate the safety and efficacy of anti-angiogenesis medicines for the treatment of advanced STS, we conducted a meta-analysis.

Methods: We systematically searched PubMed, Web of Science, Cochrane Library, Excerpta Medica Database, and China National Knowledge Infrastructure from inception to September 5, 2019. And finally, nine studies with 1,230 patients were included after rigorous screening. The endpoints included objective response rate (ORR), disease control rate (DCR), progression-free survival (PFS), overall survival (OS), and adverse reactions (AEs). And we analyzed them with the risk ratio (RR) or the hazard ratio (HR). Moreover, heterogeneity and sensitivity were also analyzed.

Results: The pooled data showed that anti-angiogenesis medicines significantly benefited ORR [risk ratio $(\mathrm{RR})=2.16,95 \% \mathrm{CI}: 1.47-3.17, \mathrm{P}<0.001$ ], and DCR $(\mathrm{RR}=1.68,95 \% \mathrm{CI}: 1.49-1.89, \mathrm{P}<0.001)$, respectively, and improved the PFS (HR =0.50, 95\% CI: 0.41-0.59, $\mathrm{P}<0.001$ ), and OS (HR =0.77, 95\% CI: 0.63-0.95, $\mathrm{P}<0.05)$, respectively. However, as for grade III or higher acute AEs, the antiangiogenic treatment increased morbidities of hypertension ( $\mathrm{RR}=4.88, \mathrm{P}<0.005)$, and diarrhea $(\mathrm{RR}=3.98, \mathrm{P}=0.021)$ compared to the control group. And no significant difference was found in bone marrow inhibition, vomiting, anemia, and mucositis.

Conclusions: Anti-angiogenesis treatments are beneficial in short-term efficacy and long-term survival for the recurrence and metastasis of STS, and the AEs are tolerable.
\end{abstract}

Keywords: Soft tissue sarcoma (STS); anti-angiogenesis medicines; meta-analysis

Submitted Nov 03, 2019. Accepted for publication Jan 07, 2020.

doi: $10.21037 /$ tcr.2020.01.47

View this article at: http://dx.doi.org/10.21037/tcr.2020.01.47

\section{Introduction}

Soft tissue sarcomas (STSs), a series of mesenchymal original malignant diseases, account for approximately $1 \%$ of human tumors (1). According to the World Health Organization (WHO), STSs include more than 70 subtypes (2), and the common subtypes of them are pleomorphic undifferentiated sarcoma, liposarcoma, leiomyosarcoma, synovial sarcoma, angiosarcoma. Surgery-based treatment might be performed to treat early-stage of STS, but the prognosis of STS remains poor, regardless of surgery, radiotherapy, and chemotherapy. On the other hand, Chemotherapy is one of the standard treatments for the advanced stage of STS. Doxorubicin $(\mathrm{ADM})$ alone or in combination with ifosfamide $(\mathrm{ADM}+\mathrm{I}-\mathrm{FO})$ is the first-line chemotherapy regimen for advanced STS. Other medicines, such as dacarbazine, 
epirubicin, and I-FO, are also used as single agent or in anthracycline-based combination therapy (3). However, the prognosis of metastatic STS still remains poor, and the 2-year median survival rate and median survival time are $38 \%$ and 18 months, respectively (4). Moreover, Folkman proposed the theory that tumor growth depends on angiogenesis (5). Angiogenesis is one of the characteristics of malignancies. This process promotes the formation of new blood vessels, resulting in the uncontrolled growth of tumors (6). Therefore, except chemotherapy, the anti-angiogenesis of tumor was considered to be researched in recent decade, marked a watershed treatment for the target therapy in STS.

The binding of a pair of key angiogenic factors, namely, vascular endothelial growth factor (VEGF) and VEGF receptor (VEGFR), is one of the key steps in the proliferation of endothelial cell, and formation of new blood vessels. Moreover, VEGF and VEGFR are highly expressed in the tumor vascular endothelial cells than in the normal vascular endothelial cells. Thus, although antiVEGF does not damage directly the tumor cells, the VEGF and VEGFR of the tumor blood vessels could be utilized as a target for tumor-directed therapy with high specificity. In addition, the activation of platelet-derived growth factor (PDGF) and its receptor (PDGFR) could induce the formation and stabilization of the outer membrane, and vascular smooth muscle of the tumor. And the insulinlike growth factor (IGF), and fibroblast growth factors (FGF) also function in tumor angiogenesis (7). Therefore, anti-angiogenesis medicines including the monoclonal antibodies, endothelial cell inhibitors, and tyrosine kinase inhibitors, could interrupt these angiogenesis-related regulatory signaling pathways to prevent angiogenesis.

Multiple clinical trials of anti-angiogenesis medicines have been performed worldwide, and various results have been obtained. Herein, these studies were presented, and the efficacy and safety of the anti-angiogenesis medicines in adult patients with advanced STS were analyzed. And we got some meaningful conclusions.

\section{Methods}

\section{Literature search}

PubMed, Web of Science, Cochrane Library, Excerpta Medica Database, and China National Knowledge Infrastructure (CNKI) were searched for articles related to anti-angiogenesis, which published from the inception of each database up to September 5, 2019. All articles were written in English or Chinese. The keywords used in the searches were "bevacizumab", "olaratumab", "endostar", "pazopanib”, "anlotinib”, "regorafenib”, "apatinib”, and "soft tissue sarcoma". A total of 2,305 papers were selected. Finally, nine studies with 1,230 patients were included after screening for the final analysis.

\section{Criteria for inclusion and exclusion}

Inclusion criteria: (I) randomized controlled trial (RCT); (II) patients with advanced or metastatic STS diagnosed and confirmed by cytology and pathology; (III) the main intervention of the experimental group was antiangiogenesis medicines based treatment; and (IV) one of the following results should be reported: objective response rate (ORR), disease control rate (DCR), progression-free survival (PFS), overall survival (OS), and adverse reactions (AEs).

Exclusion criteria: (I) non-RCT; (II) non-prospective phase II/III RCTs; (III) duplicate published data; (IV) number of cases $<40$ or patients aged below 18 years; and (V) endpoint-indicators cannot be merged.

\section{Data extraction and quality assessment}

The extracted data included authors, year of publication, treatment regimens, number of patients, intervention measures, endpoint-indicators, subtype of STSs. We used the Cochrane bias risk assessment criteria to evaluate the methodological quality of the RCTs (8). This assessment was based on the risk of bias for each important endpoint included in the trials, including blinding, the adequacy of the generation of allocation sequence, allocation concealment, and presence of incomplete endpoint data, selective endpoint, or other sources of bias.

\section{Statistical analysis}

Data were analyzed by using the Stata 12.0 statistical software. The dichotomous data of the ORR, DCR and AEs were analyzed based on the risk ratio (RR), whereas the survival data, including PFS and OS, were calculated by using the hazard ratio (HR). The $95 \%$ confidence interval (CI) was used to estimate the uncertainty, and $\mathrm{P}$ value $<0.05$ was considered statistically significant. Heterogeneity among the included RCTs was evaluated by using the $\mathrm{P}$ value and the inconsistency statistic $\left(I^{2}\right)$ statistics. Generally, 


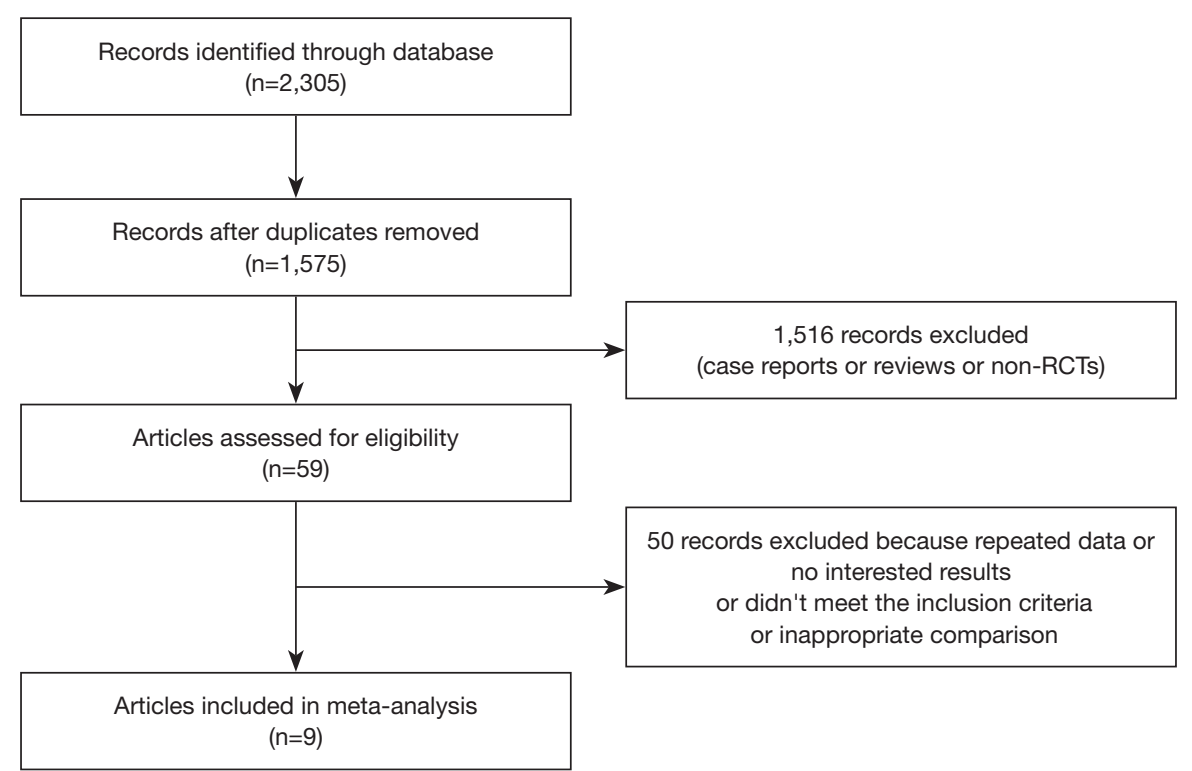

Figure 1 Flow chart of the search process for the meta-analysis.

heterogeneity was considered high for $I^{2}$ values over $50 \%$, which was brought into clinical consideration. Finally, a simple assessment was conducted through sensitivity analysis to verify the credibility of our research.

\section{Results}

\section{Search results and quality evaluation}

According to the PRISMA diagram (Figure 1), 2,305 relevant papers were obtained from the five aforementioned databases. After deleting the duplicates, 1,575 papers remained. And we searched RCTs by reading titles and abstracts among them. Finally, nine trials met the inclusion criteria, and were available for analysis. The quality of each trial was evaluated by using the Cochrane bias risk assessment criteria (Figure 2). The basic information of the included trials was listed in Table 1.

\section{Short-term efficacy: ORR and DCR}

Eight studies evaluated the short-term efficacy of antiangiogenesis medicines in patients with advanced STS. Significantly higher ORR (RR $=2.16,95 \%$ CI: $1.47-3.17$, $\mathrm{P}<0.001)$ and DCR ( RR $=1.68,95 \%$ CI: $1.49-1.89$, $\mathrm{P}<0.001)$ were observed in the study group than those in the control group (Figures 3,4). The heterogeneity among studies was low in the ORR $\left(I^{2}=33.6 \%, \mathrm{P}=0.160\right)$, but high in DCR $\left.I^{2}=68.3 \%, \mathrm{P}=0.002\right)$. Hence, a subgroup analysis of DCR was conducted in accordance with the classification of the anti-angiogenesis medicines. And the subgroup analysis showed that endothelial cell inhibitors $(\mathrm{RR}=2.26,95 \% \mathrm{CI}$ : 1.01-5.05) and tyrosine kinase inhibitors ( $\mathrm{RR}=1.93,95 \%$ CI: 1.65-2.26) were better than monoclonal antibodies (RR $=1.16,95 \%$ CI: $0.98-1.39)$ in DCR (Figure 5).

\section{Long-term efficacy: PFS and OS}

Some trials analyzed PFS and OS, respectively, and indicated that the antiangiogenic treatment group had significantly prolonged PFS ( $\mathrm{HR}=0.50,95 \%$ CI: $0.41-$ $0.59, \mathrm{P}<0.001)$ and $\mathrm{OS}(\mathrm{HR}=0.77,95 \% \mathrm{CI}: 0.63-0.95$, $\mathrm{P}<0.05$ ) compared with the control group (Figures 6,7). Unfortunately, the heterogeneity was high among the trials.

\section{AEs}

We analyzed acute grade III or higher AEs, such as myelosuppression, vomiting, hypertension, anemia, diarrhea, and mucositis. Results showed that the study group had significantly higher incidences of hypertension $(\mathrm{RR}=4.88, \mathrm{P}<0.005)$ and diarrhea $(\mathrm{RR}=3.98, \mathrm{P}=0.021)$, whereas no difference in the myelosuppression, vomiting, anemia and mucositis compared with the control group (Table 2). 


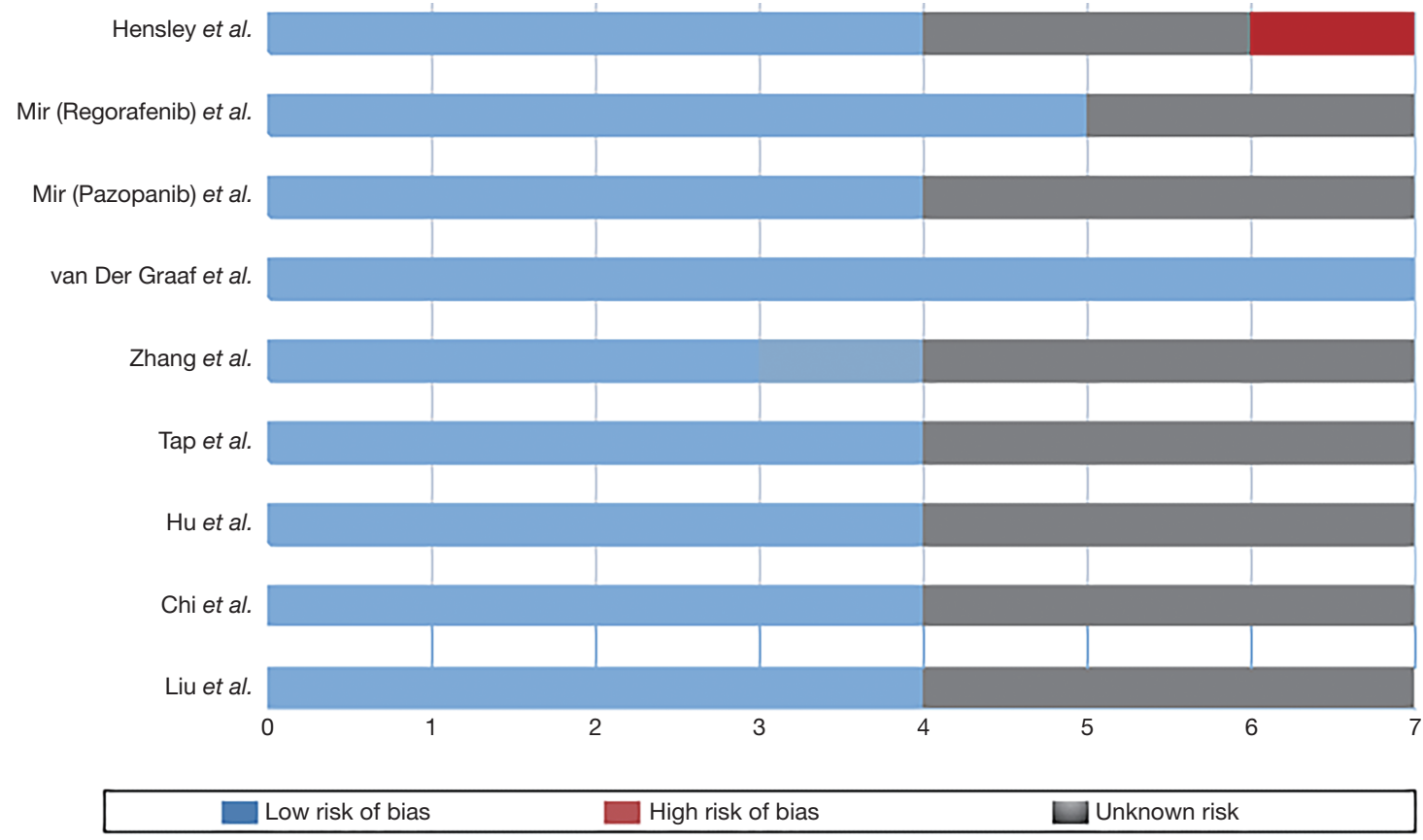

Figure 2 The quality assessment of each trial.

\section{Publication bias assessment and sensitivity analyses}

A statistical evaluation of the publication bias was not performed because of the limited number of clinical studies in our meta-analysis $(\mathrm{n}<10)$. For sensitivity analyses, the endpoint-indicators were analyzed after excluding each article (Table 3). Similar results were obtained for ORR and DCR, which showed that the sensitivity was relatively low, and our conclusions were reliable. However, PFS changed after van der Graaf et al.'s study (12) was excluded, whereas the OS changed considerably after Tap et al.'s study (14) was excluded.

\section{Discussion}

STS is a large class of malignant tumors with distinct heterogeneity in the occurrence, transformed cell types, and histopathological features, and some are in the advanced stage at the time of diagnosis, and it is difficult to treat patients with advanced STS. Fouad and Aanei elucidated the major characteristics of the tumors, namely, selective growth and proliferative advantage, altered stress response favoring OS, vascularization, invasion and metastasis, metabolic rewiring, abetting microenvironment, and immune modulation (6). It clearly indicated the significance of angiogenesis in tumors, and the great influence of the blood supply on the poor prognosis of the tumor. In recent years, several anti-angiogenesis medicines have been proved effective in multiple tumors with acceptable AEs.

The anti-angiogenesis medicines mainly include tyrosine kinase inhibitors, monoclonal antibodies, and endothelial cell inhibitors. Tyrosine kinase inhibitors such as pazopanib (target: VEGFR, PDGFR, and c-Kit), apatinib (target: VEGF and VEGFR), regorafenib (target: VEGFR, PDGFR, FGFR, and c-Kit), and anlotinib (target: VEGFR and c-Kit). Recent studies have shown that the VEGF/ VEGFR-targeted therapy displayed anti-angiogenesis and immune-supportive effects, because VEGF suppressed tumor immunity by inhibiting the maturation of dendritic cells and inducing immunosuppressive cells such as regulatory $\mathrm{T}$ cells, tumor-associated macrophages, and myeloid-derived suppressor cells (18). Monoclonal antibodies are also used as the antiangiogenic therapy for tumors. Olaratumab is a humanized PDGFR$\alpha \mathrm{Ig}$ G1 monoclonal antibody that specifically binds to PDGFR- $\alpha$, and blocks its binding to PDGF to inhibit receptor activation. Bevacizumab is a recombinant humanized monoclonal antibody that binds to VEGF and blocks its biological activity, and it's the first antitumor angiogenesis medicine approved by the US Food and Drug Administration. Anti-angiogenesis medicines also include 
Table 1 Basic information regarding the studies

\begin{tabular}{|c|c|c|c|c|c|c|c|}
\hline Study & Year & $\begin{array}{l}\text { Antiangiogenic } \\
\text { drug }\end{array}$ & Cases & Treatment arms & End points & Tumor type & $\begin{array}{l}\text { Line of } \\
\text { treatment }\end{array}$ \\
\hline \multirow[t]{2}{*}{$\begin{array}{l}\text { Hensley } \\
\text { et al. (9) }\end{array}$} & \multirow[t]{2}{*}{2015} & \multirow[t]{2}{*}{ Bevacizumab } & 53 & $\begin{array}{c}\text { Gemcitabine-docetaxel + } \\
\text { bevacizumab }\end{array}$ & \multirow[t]{2}{*}{$\begin{array}{l}\text { PFS, OS, ORR, } \\
\text { AES }\end{array}$} & \multirow[t]{2}{*}{$\begin{array}{c}\text { Uterine } \\
\text { leiomyosarcoma }\end{array}$} & \multirow[t]{2}{*}{ First-line } \\
\hline & & & 54 & $\begin{array}{c}\text { Gemcitabine-docetaxel + } \\
\text { placebo }\end{array}$ & & & \\
\hline Mir et al. (10) & 2016 & Regorafenib & 92 & Placebo & $\begin{array}{l}\text { PFS, OS, CR, } \\
\text { PR, SD, AEs }\end{array}$ & Unspecified & Non first-line \\
\hline \multirow[t]{2}{*}{ Mir et al. (11) } & \multirow[t]{2}{*}{2016} & \multirow[t]{2}{*}{ Pazopanib } & 40 & $\begin{array}{c}\text { Pazopanib + best supportive } \\
\text { care }\end{array}$ & \multirow[t]{2}{*}{$\begin{array}{l}\text { PFS, OS, SD, } \\
\text { PD, AEs }\end{array}$} & \multirow[t]{2}{*}{$\begin{array}{l}\text { Gastrointestinal } \\
\text { stromal tumours }\end{array}$} & \multirow[t]{2}{*}{ Unknown } \\
\hline & & & 41 & Best supportive care & & & \\
\hline $\begin{array}{l}\text { Zhang } \\
\text { et al. (13) }\end{array}$ & 2018 & Endostar & 26 & Gemcitabine and docetaxel & $\begin{array}{c}\text { PFS, OS, CR, } \\
\text { PR, SD, ORR, } \\
\text { DCR, AEs }\end{array}$ & Unspecified & Non first-line \\
\hline \multirow[t]{2}{*}{ Tap et al. (14) } & \multirow[t]{2}{*}{2016} & \multirow[t]{2}{*}{ Olaratumab } & 64 & Olaratumab + doxorubicin & \multirow{2}{*}{$\begin{array}{l}\text { PFS, OS, ORR, } \\
\text { AES }\end{array}$} & \multirow[t]{2}{*}{ Unspecified } & \multirow[t]{2}{*}{ Unknown } \\
\hline & & & 65 & Doxorubicin & & & \\
\hline Hu et al. (15) & 2018 & Apatinib & 21 & Apatinib + AIM & CR, PR, SD, & Unspecified & Non first-line \\
\hline & & & 21 & AIM & ORR, DCR, AEs & & \\
\hline Chi et al. (16) & 2018 & Anlotinib & 158 & Anlotinib & PFS, ORR, DCR, & Unspecified & Non first-line \\
\hline
\end{tabular}

PFS, progression-free survival; OS, overall survival; ORR, objective response rate; DCR, disease control rate; AEs, adverse events; CR, complete response; PR, partial response; SD, stable disease; AIM, adriamycin, ifosfamide, mesna.

recombinant endostatin. Endostar, an injectable antitumor vascular targeting medicine independently developed in China, could significantly inhibit the proliferation and promote the apoptosis of vascular endothelial cells, block the growth of tumor blood vessel, and inhibit the growth of tumor (19).

In this meta-analysis, the short-term efficacy of patients with advanced STS was significantly improved in the antiangiogenesis medicine group ( $\mathrm{RR}=2.16,95 \% \mathrm{CI}: 1.47$ 3.17 in ORR; RR $=1.68,95 \%$ CI: $1.49-1.89$ in DCR). A subgroup analysis was conducted, and results showed that compared with the monoclonal antibodies, the inhibitors of endothelial cell and tyrosine kinase significantly presented better benefits in DCR. Moreover, the anti-angiogenesis medicines significantly improved PFS and OS compared with the control group (HR $=0.50,95 \%$ CI: $0.41-0.59$ in PFS; HR $=0.77,95 \%$ CI: $0.63-0.95$ in OS). In addition, the heterogeneity of these endpoint-indicators was examined. The sensitivity analysis found that removing van der Graaf et al.'s study (12) influenced the PFS (HR $=0.78,95 \%$ CI: 0.61-1.00), whereas removing Tap et al.'s study (14) influenced the OS (HR $=0.90,95 \%$ CI: 0.71-1.13). Three trials $(10,16,17)$ showed significant benefits in PFS or OS, but they cannot be used in the analysis of PFS or OS without HR and $95 \%$ CI. Therefore, only parts of the studies were included in the analysis of long-term survival benefits, and the high heterogeneity and sensitivity of PFS and OS may be related to this limitation.

The failure of Hensley et al.'s study (9) might be attributed to the focus on the single histology (leiomyosarcoma) of 


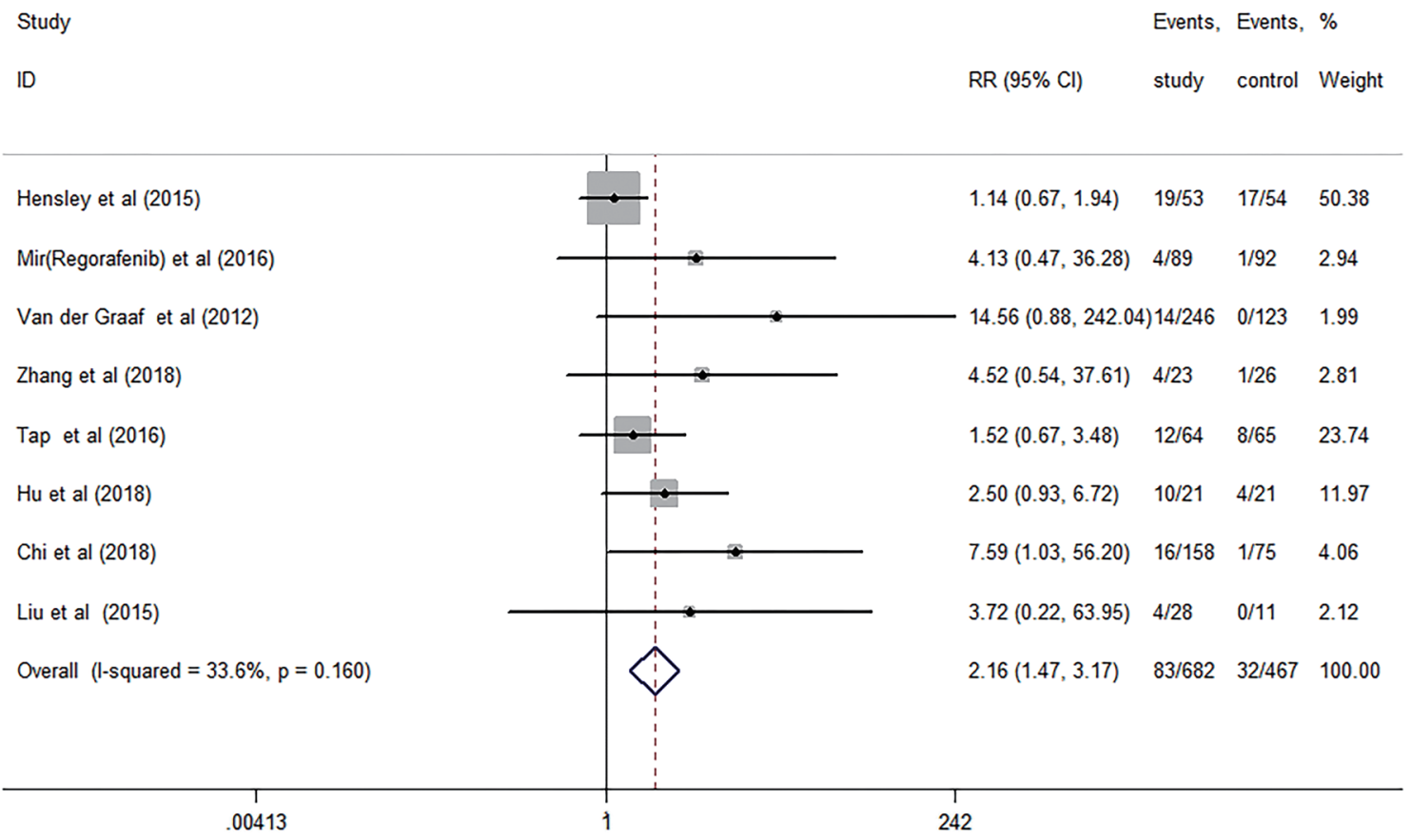

Figure 3 Forest plot of the risk ratio of objective response rate (ORR) between anti-angiogenesis treatments and control group. The fixedeffects model was used in the analysis. RR, risk ratio; CI, confidence interval.

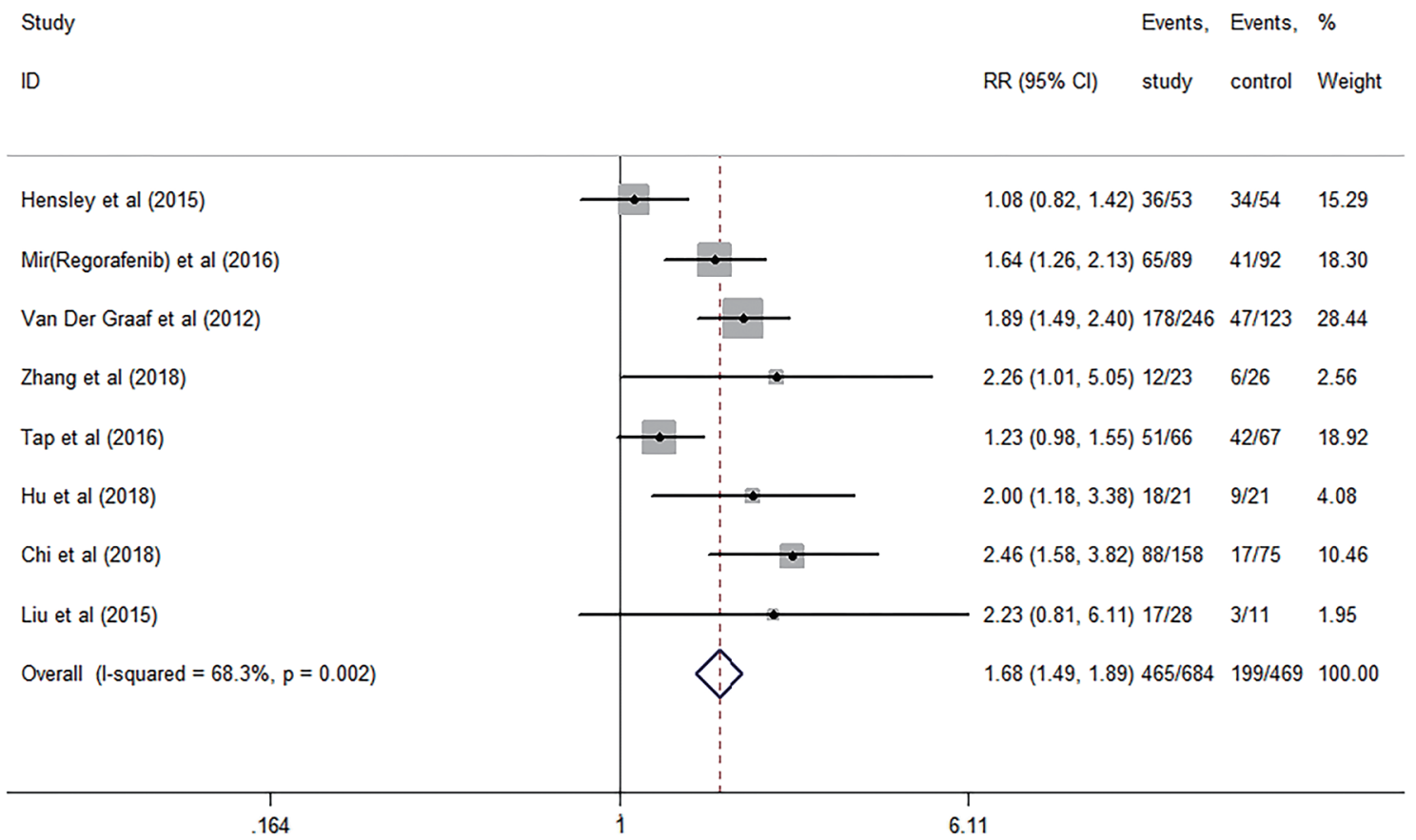

Figure 4 Forest plot of the risk ratio of disease control rate (DCR) between anti-angiogenesis treatments and control group. The fixedeffects model was used in the analysis. RR, risk ratio; CI, confidence interval. 
Study

ID
Events, Events, \%

$\mathrm{RR}(95 \% \mathrm{Cl})$ study control Weight

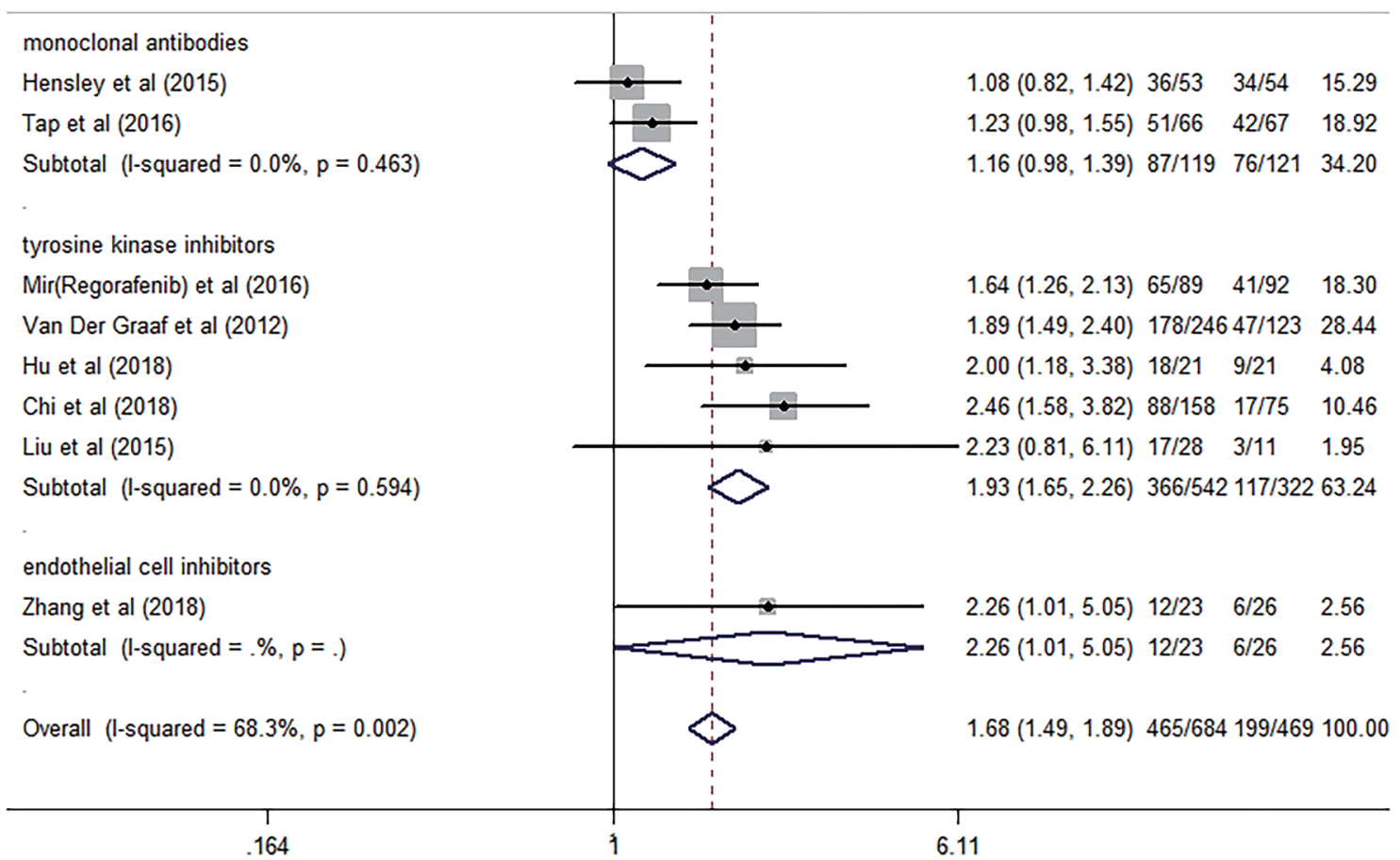

Figure 5 Forest plot of the risk ratio of subgroup analysis of disease control rate (DCR) according to the classification of anti-angiogenesis medicines. The fixed-effects model was used in the analysis. RR, risk ratio; CI, confidence interval.

Study

ID
$\%$

HR $(95 \% \mathrm{Cl}) \quad$ Weight

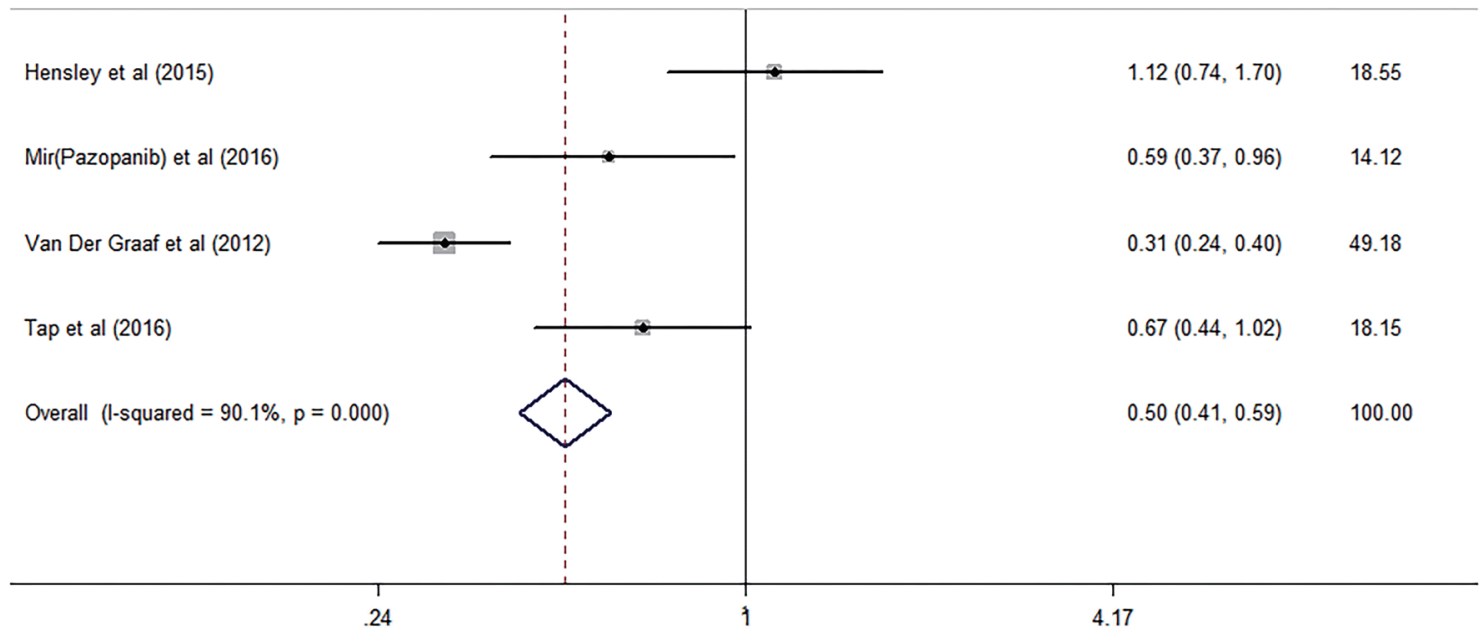

Figure 6 Forest plot of the hazard ratio of progression-free survival (PFS) between anti-angiogenesis treatments and control group. The fixed-effects model was used in the analysis. HR, hazard ratio; CI, confidence interval. 


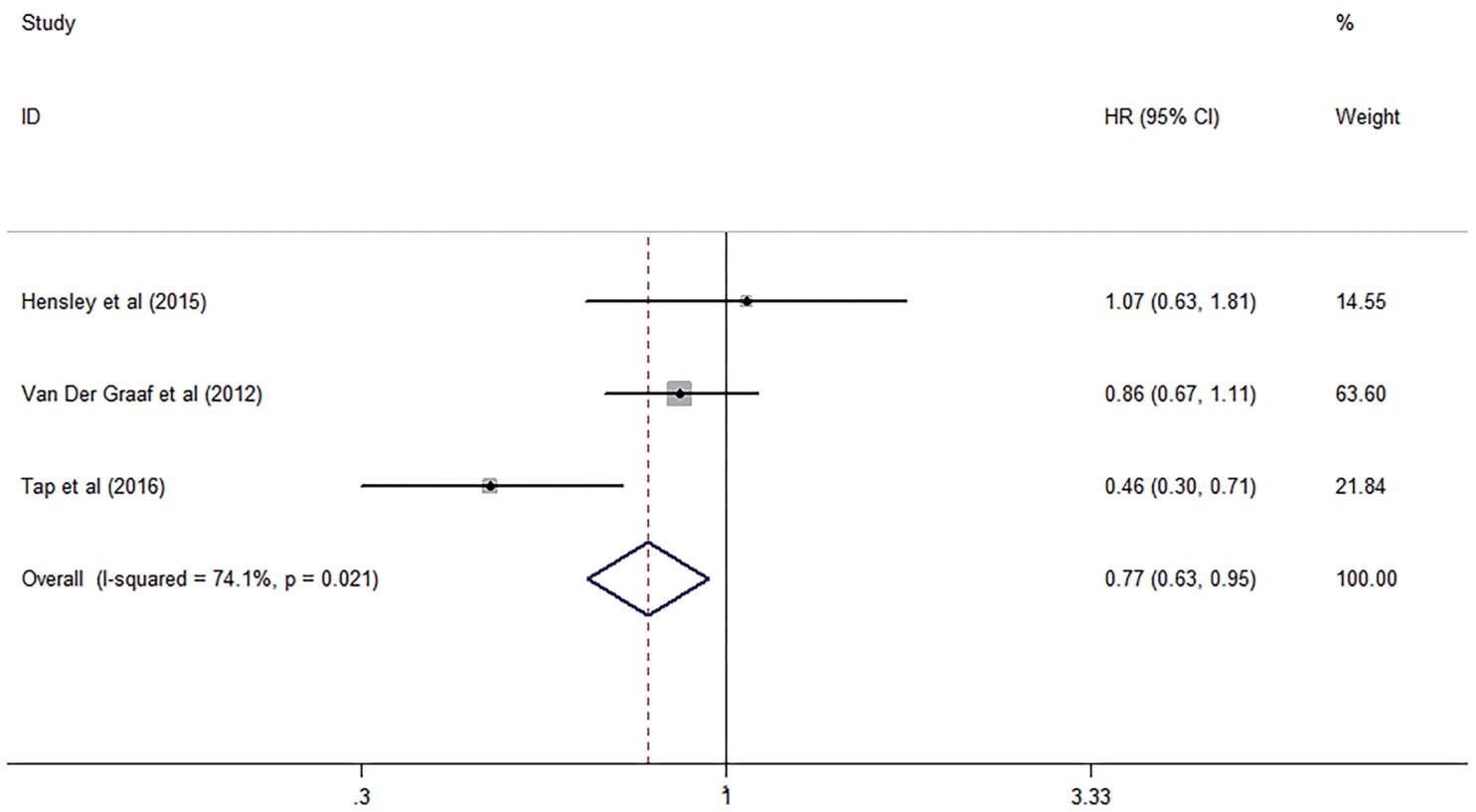

Figure 7 Forest plot of the hazard ratio of overall survival (OS) between anti-angiogenesis treatments and control group. The fixed-effects model was used in the analysis. HR, hazard ratio; CI, confidence interval.

Table 2 Adverse reactions of the antiangiogenic treatment group vs. the control group

\begin{tabular}{|c|c|c|c|c|c|}
\hline Adverse reaction & Study No. & $\mathrm{RR}$ & \multicolumn{2}{|c|}{$95 \% \mathrm{Cl}$} & $P$ value \\
\hline Hypertension & 6 & 4.88 & 2.3 & 10.33 & $<0.005$ \\
\hline Myelosuppression & 5 & 1.36 & 0.98 & 1.89 & 0.067 \\
\hline Vomiting & 3 & 3.66 & 0.79 & 16.93 & 0.097 \\
\hline Diarrhoea & 3 & 3.98 & 1.23 & 12.87 & 0.021 \\
\hline Mucositis & 3 & 2.17 & 0.67 & 7.04 & 0.196 \\
\hline
\end{tabular}

$\mathrm{RR}$, risk ratio; $\mathrm{Cl}$, confidence interval.

a single anatomical site (uterus). This result might be different from other STSs because different sarcomas might have different medicine sensitivities. Bevacizumab is shown to be effective in angiosarcoma metastasis, locally advanced angiosarcoma, and epithelioid hemangioendotheliomas (20). Only Hensley et al.'s study was applied in the first-line therapy. Thus, the suitability of anti-angiogenesis medicines for first-line therapy in advanced STS should also be considered. The clinical result of pazopanib indicated a significant survival benefit compared with standard chemotherapy in different STS types (21). This result might be related to the multiple targets and high activity of pazopanib. Furthermore, some studies have reported that the effect of the combination of anti-angiogenesis medicines with chemotherapy is related to the timing of application, but the time window for combination remains unclear. Studies included in this meta-analysis were concurrently administered. Therefore, a certain conclusion about the schedule of administration in advanced STS cannot be drawn, and more studies should be conducted to better optimize the doubt.

Majority of the trials reported that the AEs of antiangiogenesis medicines were tolerable. The anti-angiogenesis medicine group showed no significant difference in 


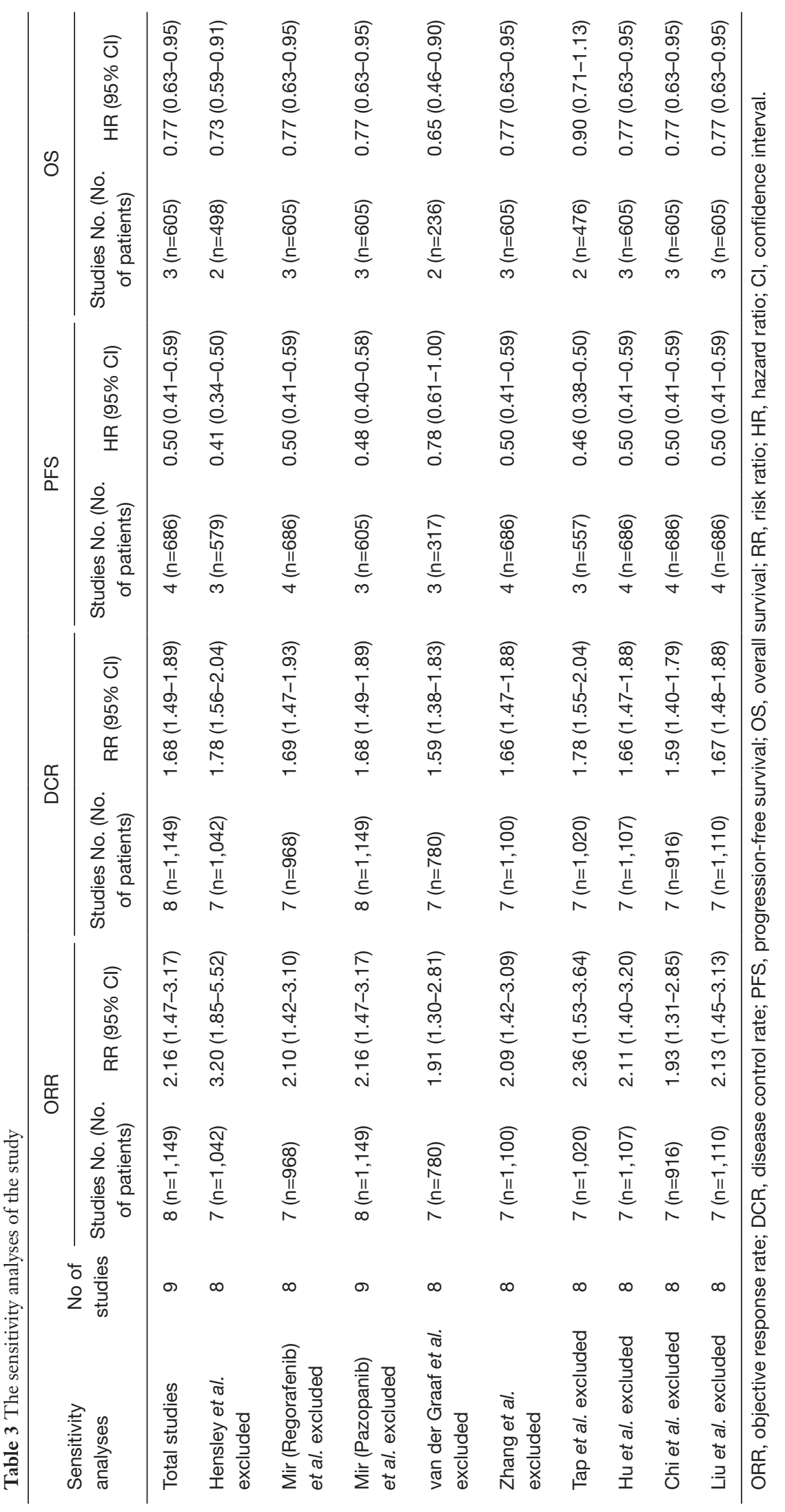


myelosuppression, mucositis, anemia and vomiting compared with the control group, but the former experienced induced higher incidence of hypertension and diarrhea. The specific mechanisms of AEs are still unknown. However, some studies have indicated that VEGFR inhibitors reduce the production of prostacyclin and NO by impairing the VEGFR of endothelial cells, resulting in hypertension (22). The cause of diarrhea may be related to the medicine-induced pancreatic exocrine dysfunction. Moreover, it might exist some kinds of off-target toxicities that didn't related to the anti-angiogenesis target treatments.

This meta-analysis had several limitations. In first place, the data of the trials was not sufficient for an in-depth analysis of PFS and OS. Additional clinical data is necessary for the survival benefit of anti-angiogenesis treatments. Three studies without HR and $95 \% \mathrm{CI}$ in the analysis of PFS and OS were excluded, and this exclusion may have a certain effect on our endpoint-indicators. Secondly, a subgroup analysis of medicines and doses couldn't be performed because of the limited number of studies. Moreover, two of the included studies $(9,11)$ were focused on a specific soft tissue tumor, and whether the anti-angiogenesis treatments work in other STSs or not was uncertain.

Although anti-angiogenesis treatment can suppress tumor blood vessels, its antitumor activity remains low, and suffered from resistance easily. Therefore, the combination of different treatment methods is a promising approach in cancer therapy.

The decreased blood supply and increased oxygen consumption cause the tumor-specific microenvironment hypoxia. This condition impairs the efficacy of radiotherapy and results in local recurrence and distant metastasis. The optimized application of antiangiogenic therapy can normalize the tumor blood vessels, and upregulate the oxygen supply. Thus, the combination of antiangiogenic therapy with radiotherapy should be an ideal local and systemic treatment. Moreover, this combination can enhance the killing power to endothelial cells, and inhibit tumor metastasis to achieve better therapeutic effects (23). However, few studies in this field for STS have been conducted.

In addition, most of the included trials have shown that anti-angiogenesis therapy combined with chemotherapy can also benefit the patients' survival. The combined therapy has also been extensively studied in other solid tumors, and has yielded positive results, although AEs have also increased accordingly. Although many trails have confirmed the synergistic effect of anti-angiogenesis medicines combined with chemotherapy (24), some trials showed no enhancement of the survival benefit by using this combination therapy in comparison with chemotherapy alone. This suggested that a $1+1>2$ effect might be more likely to be achieved only if the anti-angiogenesis medicines are combined with the chemotherapy in the optimal treatment regimen and the optimal timing (25). Therefore, further exploration should be carried out based on the specific mechanism of combined application, timing, and side effects in the future.

\section{Conclusions}

Anti-angiogenesis medicines have shown short-term and long-term benefits for advanced STS in our study, indicating its promising role as an anticancer medicine, with increased incidence of some tolerable AEs. In addition, although long-term efficacy has shown overall benefits, the reason for the failure of bevacizumab to treat leiomyosarcoma is worthy of attention. Further RCTs are warranted to update our meta-analysis, and investigate the role of anti-angiogenesis medicines in patients with STS.

\section{Acknowledgments}

Funding: None.

\section{Footnote}

Conflicts of Interest: All authors have completed the ICMJE uniform disclosure form (available at http://dx.doi. org/10.21037/tcr.2020.01.47). The authors have no conflicts of interest to declare.

Ethical Statement: The authors are accountable for all aspects of the work in ensuring that questions related to the accuracy or integrity of any part of the work are appropriately investigated and resolved.

Open Access Statement: This is an Open Access article distributed in accordance with the Creative Commons Attribution-NonCommercial-NoDerivs 4.0 International License (CC BY-NC-ND 4.0), which permits the noncommercial replication and distribution of the article with the strict proviso that no changes or edits are made and the original work is properly cited (including links to both the formal publication through the relevant DOI and the license). See: https://creativecommons.org/licenses/by-nc-nd/4.0/. 


\section{References}

1. Smith SR. Rehabilitation strategies and outcomes of the sarcoma patient. Phys Med Rehabil Clin N Am 2017;28:171-80.

2. Bourcier K, Cesne AL, Tselikas L, et al. Basic knowledge in soft tissue sarcoma. Cardiovasc Intervent Radiol 2019;42:1255-61.

3. Kawai A, Yonemori K, Takahashi S, et al. Systemic Therapy for Soft Tissue Sarcoma: Proposals for the Optimal Use of Pazopanib, Trabectedin, and Eribulin. Adv Ther 2017;34:1556-71.

4. Hall F, Villalobos V, Wilky B. Future directions in soft tissue sarcoma treatment. Curr Probl Cancer 2019;43:300-7.

5. Lugano R, Ramachandran M, Dimberg A. Tumor angiogenesis: causes, consequences, challenges and opportunities. Cell Mol Life Sci 2019. [Epub ahead of print].

6. Fouad YA, Aanei C. Revisiting the hallmarks of cancer. Am J Cancer Res 2017;7:1016-36.

7. Gao T, Fan ZF, Fang ZW. Advances in molecular targeted therapy for soft tissue sarcomas. Chin J Clin Oncol 2017;44:7-13.

8. Higgins J, Green S. Cochrane handbook for systematic reviews of interventions. The Cochrane Collaboration Version 5.10. 2011. [Accessed September 5, 2018]. Available online: http://www.cochrane-handbook.org/

9. Hensley ML, Miller A, O'Malley DM, et al. Randomized phase III trial of gemcitabine plus docetaxel plus bevacizumab or placebo as first-line treatment for metastatic uterine leiomyosarcoma: an NRG oncology/gynecologic oncology group study. J Clin Oncol 2015;33:1180-5.

10. Mir O, Brodowicz T, Italiano A, et al. Safety and efficacy of regorafenib in patients with advanced soft tissue sarcoma (REGOSARC): a randomised, double-blind, placebocontrolled, phase 2 trial. Lancet Oncol 2016;17:1732-42.

11. Mir O, Cropet C, Toulmonde M, et al. Pazopanib plus best supportive care versus best supportive care alone in advanced gastrointestinal stromal tumours resistant to imatinib and sunitinib (PAZOGIST): a randomised, multicentre, openlabel phase 2 trial. Lancet Oncol 2016;17:632-41.

12. van der Graaf WT, Blay JY, Chawla SP, et al. Pazopanib for metastatic soft-tissue sarcoma (PALETTE): a randomised, double-blind, placebo-controlled phase 3 trial. Lancet 2012;379:1879-86.

13. Zhang DB, Kang YX, Guo YZ. Efficacy and safety of endostar combined with gemcitabine and docetaxel in advanced soft tissue sarcomas. Journal of Modern Oncology 2018;26:769-72.

14. Tap WD, Jones RL, Van Tine BA, et al. Olaratumab and doxorubicin versus doxorubicin alone for treatment of softtissue sarcoma: an open-label phase $1 \mathrm{~b}$ and randomised phase 2 trial. Lancet 2016;388:488-97.

15. Hu MQ, Gao K. Observation of clinical effect of apatinib combined with AIM regimen in the treatment of advanced soft tissue sarcoma. Clinical Medication Journal 2018;16:62-5.

16. Chi Y, Yao Y, Wang S, et al. Anlotinib for metastasis soft tissue sarcoma: a randomized, double-blind, placebocontrolled and multi-centered clinical trial. J Clin Oncol 2018;36:abstr 11503.

17. Liu JY, Fan ZF, Li S, et al. Anlotinib hydrochloride capsules for advanced soft tissue sarcoma: single-center data analysis of a stage II multicenter clinical trial. Chin J Clin Oncol 2018;45:1066-70.

18. Tamura R, Tanaka T, Akasaki Y, et al. The role of vascular endothelial growth factor in the hypoxic and immunosuppressive tumor microenvironment: perspectives for therapeutic implications. Med Oncol 2019;37:2.

19. Han NN, Jiang QY, Li L. Advance in the Clinical Mechanism of Recombinant Human Endostatin. Xian Dai Sheng Wu Yi Xue Jin Zhan 2019;19:2993-5.

20. Yin QL, Wu D, Yu YY. Progression in anti-angiogenesis agents for soft tissue sarcoma. Chin Clin Oncol 2017;22:839-44.

21. Grünwald V, Kunitz A, Schuler MK, et al. Randomized comparison of pazopanib (PAZ) and doxorubicin (DOX) in the first line treatment of metastatic soft tissue sarcoma (STS) in elderly patients (pts): Results of a phase II study (EPAZ). J Clin Oncol 2018;36:abstr 11506.

22. Touyz RM, Lang NN, Herrmann J, et al. Recent advances in hypertension and cardiovascular toxicities with vascular endothelial growth factor (VEGF) inhibition. Hypertension 2017;70:220-6.

23. Goedegebuure RSA, de Klerk LK, Bass AJ, et al. Combining Radiotherapy With Anti-angiogenic Therapy and Immunotherapy; A Therapeutic Triad for Cancer? Front Immunol 2019;9:3107.

24. Ferrarelli LK. Tumors direct vessels to feed growth. Sci Signal 2017. doi: 10.1126/scisignal.aam9091.

25. Wu F, Chen FW, Liu FW, et al. Observation of the antitumor effect of endostar combined with taxel under different administration sequences. The Practical Journal of Cancer 2018;33:697-9.

Cite this article as: Wang Y, Dai X, Zhu Y. Efficacy and safety of anti-angiogenesis medicines for advanced soft tissue sarcoma: a meta-analysis. Transl Cancer Res 2020;9(3):1536-1546. doi: 10.21037/tcr.2020.01.47 